\title{
High-fat diets impair conditional discrimination learning in rats
}

\author{
GORDON WINOCUR \\ Rotman Research Institute, Toronto, Ontario, Canada \\ and Trent University, Peterborough, Ontario, Canada \\ and \\ CAROL E. GREENWOOD \\ University of Toronto, Toronto, Ontario, Canada
}

\begin{abstract}
At the age of 1 month, four separate groups of Long-Evans rats were placed on diets varying in the amount and composition of dietary fat. High-saturated-fat diets (lard based) were either $20 \%$ or $10 \%(w / w)$ of the diet. High-polyunsaturated-fat diets (soybean oil based) were either $20 \%$ or $5 \%(\mathrm{w} / \mathrm{w})$ of the diet. A fifth group of rats consumed standard laboratory chow $(4.5 \% \mathrm{w} / \mathrm{w}$ fat). After 3 months, all the rats were tested on a conditional discrimination learning task in which they were required to associate different stimuli with different responses. Independent of the source of dietary fat, the animals fed high-fat diets $(20 \% \mathrm{w} / \mathrm{w})$ were impaired on the task. Performance of the animals fed the $10 \%$ lard diet was indistinguishable from that of the animals fed the $20 \%$ fat diets. In contrast, the rats fed $5 \%$ soybean oil (SBO) or chow diets displayed superior performance in comparison with all other groups. These data demonstrate that high-fat diets can have adverse effects on learning and memory and provide suggestive evidence that saturated fatty acids may be associated with the adverse effect.
\end{abstract}

The ability of diet to influence cognitive performance in humans or experimental animals remains controversial. Limited evidence suggests that diets inadequate in nutrients such as vitamins and minerals (for reviews see Dreyfus, 1988; Sandstead, 1986) and essential fatty acids (for review see Greenwood, McGee, \& Dyer, 1989) may impair cognitive performance. Inadequate diets, especially if consumed over a long term, will impair metabolic pathways in the central nervous system that are dependent upon an adequate supply of nutrients (for review see Greenwood \& Craig, 1987). Thus, dietary inadequacy resulting in altered brain metabolism could result in impaired function. Of broader relevance is the question of whether normal variations in the diet, which do not include nutrient inadequacy, can influence cognitive performance.

Our interest in this area relates to the role of dietary fat intake in influencing brain function and derives from three lines of reasoning. First, most national dietary guidelines recommend a decrease in total fat content of the diet and, specifically, a reduction in saturated fatty acid intake to reduce the risk of chronic diseases including cardiovascular disease and cancer. Yet the impact of these dietary changes on central nervous tissue metabolism and

The authors wish to acknowledge the technical assistance of Bruce Scott, Susan DiFrancesco, and John Zorner. This research was supported by grants from the National Sciences and Engineering Research Council of Canada. Address correspondence to G. Winocur, Rotman Research Institute, Baycrest Centre for Geriatric Care, 3560 Bathurst St., Toronto, ON Canada, M6A 2 E1. function has not been extensively assessed. Second, results from our laboratory (Dyer \& Greenwood, 199la, 1991b, 1991c) and others (Foot, Cruz, \& Clandinin, 1982) demonstrate that brain membrane fatty acid composition responds to alterations in dietary fat content and composition. Since these changes can be observed in the developed brain, the brain may be responsive to effects of dietary fat intake, even if they are instituted relatively late in life. Third, certain animal behaviors, including pain sensitivity, thermoregulation following an amphetamine challenge (Yehuda, Leprohon-Greenwood, Dixon, \& Coscina, 1986), and feeding behavior (selection of protein and carbohydrate; McGee \& Greenwood, 1990a, $1990 \mathrm{~b})$, respond to alterations in the composition of dietary fat fed to rats.

As a consequence of these observations, we initiated a series of experiments designed to determine whether changing the composition of fat in diets fed to rats could affect their cognitive performance. In this research, rats were fed a diet high in saturated fatty acids $(20 \% \mathrm{w} / \mathrm{w}$ lard-based diet; lard) or polyunsaturated fatty acids (20\% w/w soybean-oil-based diet; SBO) and administered the following behavioral tasks-Olton's radial-arm maze, a variable-interval delayed alternation task, and the HebbWilliams maze series (Greenwood \& Winocur, 1990). In general, both groups of rats performed worse than a group fed standard laboratory chow, but the lard group was severely impaired on all measures and consistently performed worse than the SBO group. The deficit of the lardfed rats was widespread and extended to the acquisition 
of maze-learning skills, as measured by rate of improvement over the series of complex mazes, and learning the response-alternation rule in the delayed alternation task. These results indicated that the lard diet produced a generalized impairment that included poor memory for specific or episodic events as well as deficits in nonspecific learning and memory functions.

An interesting feature of these results was that the ability to acquire rule-based behaviors was compromised in rats fed a diet high in saturated fatty acids. In the present experiment, we tested the generality of this observation by comparing the effects of various diets on a test of conditional discrimination learning (CDL), in which correct response selection was governed by an explicit rule. The test was conducted in an operant chamber that was outfitted with a panel of lights and two retractable levers. On each trial, a different combination of lights was illuminated at the same time that both levers were presented. The basic rule was that illumination anywhere on the left side of the panel was the signal for pressing the left lever, whereas illumination on the right side called for a rightlever response.

An important feature of the CDL task is that it measures animals' ability to learn an associative rule independently of their ability to recall specific events. This test has been used in neuropsychological investigations to compare the effects of lesions to different brain regions (Winocur, 1991). Damage to the hippocampus, a structure known to be critically involved in memory for specific events, did not affect performance under the conditions employed in the present experiment. On the other hand, rats with lesions to the prefrontal cortex were severely impaired, indicating that performance on this task depends on the functional integrity of this region. In our previous work (Greenwood \& Winocur, 1990), lard-fed rats were reliably impaired on behavioral tests of hippocampal function and there was evidence that performance mediated by other neural structures may also have been impaired. The present study affords the opportunity to determine if cognitive functions controlled by prefrontal cortex are affected by the composition of fat in animals' diets.

Another purpose of this study was to examine the effects of varying the concentration of fat in diet. In our previous work, the level of dietary fat $(20 \% \mathrm{w} / \mathrm{w}$, or $40 \%$ of calories) was chosen to represent typical North American human consumption patterns. However, we felt it important to assess the impact of lowering the overall fat content of the diet to determine whether negative effects of diets high in saturated fatty acids can also be observed at levels lower than that observed in our previous study. In order to ensure that all the diets contained sufficient essential fatty acids (EFA) to meet the rat's requirement, $10 \%$ lard and 5\% SBO groups were included. A lower level of lard in the diet was not studied, because a $5 \%$ lard diet would be nutritionally deficient in EFA.

\section{METHOD}

\section{Subjects and Diets}

Thirty-nine experimentally naive, male Long-Evans rats were obtained from the Trent University Breeding Centre and served as subjects. When the rats were 1 month old and weighed $60-80 \mathrm{~g}$, they were randomly assigned to one of five groups-lard20 $(n=$ $7)$, lard10 $(n=8)$, SBO20 $(n=8)$, SBO5 $(n=8)$, or standard laboratory chow ( $n=8$; Ralston Purina, St. Louis, MO). The experimental diets (Table 1 ) contained $27 \mathrm{~g}$ casein, 5 g nonnutritive fiber, $2.5 \mathrm{~g}$ vitamin mixture (Teklad Test Diets, Madison, WI), $5.1 \mathrm{~g}$ Bernhart-Tomarelli mineral mixture (Bernhart \& Tomarelli, 1969), and $0.25 \mathrm{~g}$ methionine. Fat was added to the diet at the expense of cornstarch to make $100 \mathrm{~g}$. Two diets contained either $5 \%$ or $20 \%(w / w)$ soybean oil (SBO), and the other two diets contained either $10 \%$ or $20 \%$ lard (supplemented with 5\% SBO and safflower oil prior to mixing). Diet formulation of the semisynthetic diets was verified by determining protein content (micro-Kjeldahl) and fatty acid profile after mixing. Fat was extracted from the diets, and fatty acid methyl esters were prepared and analyzed by gas-liquid chromatography using flame ionization detection as described previously (Dyer \& Greenwood, 1991c). The fatty acid profile of the diets is provided in Table 2. Supplementation of the lard with SBO and safflower oil prior to mixing the diet ensured adequacy of EFA intake. A 5\% lard diet was not tested, because the addition of sufficient EFA to meet the animal's requirement of this diet would bring the fatty acid profile of the diet close to that of the 5\% SBO and would not allow for sufficient distinction between the composition of these two diets.

The rats were provided ad-lib diet and water until they were 4 months of age. At this time, they were gradually reduced, over a 2-week period, to $80 \%$ of their body weights. Throughout the experiment, food intake was adjusted in order to maintain the rats at about $80 \%$ of their normal body weights.

Table 1

Diet Composition (Grams/Kilogram Diet)

\begin{tabular}{|c|c|c|c|c|}
\hline Ingredient & $10 \%$ Lard & $20 \%$ Lard & $\begin{array}{c}5 \% \text { Soybean } \\
\text { Oil }\end{array}$ & $\begin{array}{c}20 \% \text { Soybean } \\
\text { Oil } \\
\end{array}$ \\
\hline Casein* & 270.0 & 270.0 & 270.0 & 270.0 \\
\hline Cornstarch & 501.5 & 401.5 & 551.5 & 401.5 \\
\hline Cellulose & 50.0 & 50.0 & 50.0 & 50.0 \\
\hline Vitamin $\mathbf{M i x}^{*}$ & 25.0 & 25.0 & 25.0 & 25.0 \\
\hline Mineral Mix* & 51.0 & 51.0 & 51.0 & 51.0 \\
\hline L-Methionine & 2.5 & 2.5 & 2.5 & 2.5 \\
\hline Fat & $100.0 \dagger$ & 200.0 & 50.0 & 200.0 \\
\hline Calories (kcal/g diet) & 3.99 & 4.49 & 3.74 & 4.49 \\
\hline
\end{tabular}

*Purchased from Teklad (Madison, WI). Casein was $87 \%$ protein. Composition of the vitamin (TD 67231; Musten, Peace, \& Anderson, 1974) and mineral (Bernhard \& Tomarelli, 1969) mixtures have been published elsewhere. †Lard was blended with $5 \%$ safflower and soybean oils before being blended into the diets. 
Table 2

Fatty Acid Composition of Diets (Percentage Fatty Acids per $100 \mathrm{~g}$ Diet)

\begin{tabular}{lccccc}
\hline Fatty Acid & $\begin{array}{c}\text { Laboratory } \\
\text { Chow }\end{array}$ & 10\% Lard & 20\% Lard & $\begin{array}{c}\text { 5\% Soybean } \\
\text { Oil }\end{array}$ & $\begin{array}{c}\text { 20\% Soybean } \\
\text { Oil }\end{array}$ \\
\hline C14:0 & 0.05 & 0.18 & 0.34 & 0.01 & 0.09 \\
C16:0 & 0.68 & 2.40 & 4.71 & 0.57 & 2.33 \\
C16:1 & 0.06 & 0.10 & 0.34 & & \\
C18:0 & 0.16 & 1.22 & 2.48 & 0.11 & 0.81 \\
C18:1 & 0.90 & 4.14 & 8.18 & 1.13 & 4.55 \\
C18:2n6 & 2.28 & 1.87 & 3.77 & 2.83 & 10.94 \\
C18:3n3 & 0.23 & 0.10 & 0.24 & 0.35 & 1.23 \\
C22:6n3 & 0.09 & & & & \\
C24:0 & 0.03 & & & & \\
\hline
\end{tabular}

Note - Lipid was extracted from premixed diets and analyzed for fatty acid content by gas-liquid chromatography using flame-ionization detection.

\section{Apparatus}

All testing was conducted in five identical operant chambers $(29 \times 28 \times 26 \mathrm{~cm})$, each outfitted with two retractable levers, a centrally located visual display panel, and a food well. The display panel consisted of a bank of two rows of 2 to 3.5 -cp lights, each $1 \mathrm{~cm}$ in diameter and spaced $3 \mathrm{~cm}$ apart. The food well was situated centrally, $5 \mathrm{~cm}$ below the lights and between the levers. A pellet dispenser delivered 45-mg Noyes food pellets to the food well. Three walls and the ceiling of each box were made of Plexiglas. The fourth wall, containing the levers, panel, and food well, was made of metal, and the floor consisted of steel rods spaced $1.5 \mathrm{~cm}$ apart.

All aspects of testing, including data recording, were controlled by an Apple II + microcomputer connected to each chamber.

\section{Procedure}

The animals were handled on a regular basis once food restriction had commenced. When body weights had stabilized to $80 \%$ of normal, each rat underwent an extensive three-stage training program before experimental testing began.

Stage 1. The rats received daily sessions in which they were trained to press both levers according to a continuous reinforcement (CRF) schedule. On the first day, they received some hand shaping, but on the following days, they were left to press the levers at their own rates. A rat could obtain a maximum of 160 food pellets per session, distributed equally across left and right leverpresses. When a lever was pressed 80 times, it remained in the chamber, but subsequent presses no longer delivered pellets. Each session in Stage 1 ended when 160 pellets had been delivered, or after a 30 -min time limit. The criterion for completing Stage 1 was three consecutive sessions in which the maximum number of pellets was obtained.

Stage 2. The day after criterion was reached on Stage 1, the rats advanced to Stage 2 in which they were trained to become familiar with levers retracting after reinforcement. Each daily session began with both levers present. Pressing either lever produced a food pellet, followed by the retraction of both levers for $10 \mathrm{sec}$. The levers then reappeared and remained in the chamber until a response was performed. The cycle was then repeated. Each lever could be pressed 80 times for reinforcement. When 80 pellets had been obtained with a lever, it no longer reappeared, and the session continued with only the other lever appearing and retracting. This procedure served to discourage the build-up of position preferences. The criterion for completing Stage 2 was obtaining 160 reinforcements in three consecutive 30 -min sessions.

Stage 3. The day after the criterion was reached on Stage 2, Stage 3 was instituted to train the rats to respond differentially to the presence and absence of lights on a given trial. Daily sessions consisted of 100 light and 100 no-light trials, with the order of pre- sentation randomly determined. A light trial began with illumination of all four lights and the simultaneous appearance of both levers. When a rat pressed a lever, a pellet was dispensed, the lights turned off, and the levers retracted. A 10-sec interval followed each light trial. In the light trials, the rats were permitted a maximum of $\mathbf{5 0}$ responses to each lever. When that number was reached, the lever was retracted and did not reappear in the session. In the no-light trials, both levers appeared for $10 \mathrm{sec}$ or until the rat pressed one of the levers. No pellets were provided in the no-light trials. If the rat pressed a lever during a no-light trial, a 30-sec intertrial interval followed.

A session was terminated when 200 trials (100 light and 100 nolight) were completed. The criterion for completing Stage 3 was three consecutive sessions in which the ratio of leverpresses during the light trials to leverpresses during the no-light trials was 2:1 or better.

Testing. Testing on the conditional learning task began the day after completion was reached on Stage 3. Each session began with the illumination of the top, bottom, or both lights on either the left or right side of the panel. The lights served as discriminative stimuli and were accompanied by the simultaneous appearance of both levers. The stimuli and levers were present until a response was performed.

A stimulus on the left side was the signal for pressing the left lever, and a stimulus on the right side was the signal for a rightlever response. A correct response resulted in the removal of the stimulus, the delivery of a food pellet, retraction of both levers, and a 10-sec intertrial interval. An incorrect response led to the removal of the stimulus, retraction of the levers, no reinforcement, and a 30 -sec intertrial interval.

Each session consisted of 80 trials in which the left lever was correct and 80 trials in which the right lever was correct; the order was randomly determined. One 160-trial session was administered each day for 30 days. Data were expressed as the ratio of correct to incorrect responses for each session; the higher the ratio, the superior the performance on the task. A ratio of 1 meant that the rats were unable to discriminate throughout the trial. For ease of presentation, the data for each animal were collapsed into six blocks of five sessions each, prior to statistical analysis.

\section{RESULTS}

Records were kept of the rats' progress throughout the training program. There were no group differences in reaching criterion in either Stage 1, in which the rats learned to leverpress, or in Stage 2, in which they became familiar with the retraction of levers following re- 


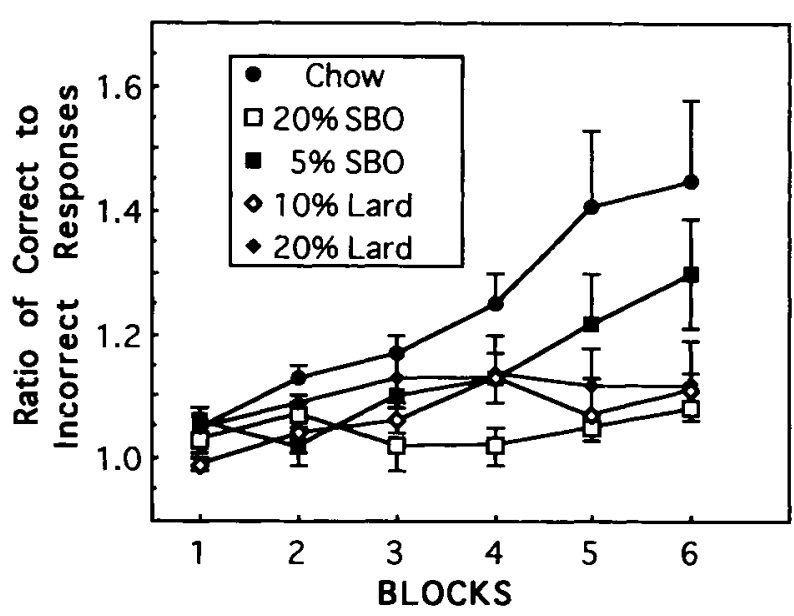

Figure 1. Mean \pm 1 standard deviation of the ratio of correct to incorrect responses on the conditional discrimination learning task. Data are presented over six blocks of 3 test days each.

sponses. In Stage 3, in which the rats were trained to relate leverpressing to the presence of a visual stimulus, the groups reached criterion after about 12 days (range 10-19) of training. The lard20 group required the greatest number of trials $(M=14.7$, range $11-19)$, but an analysis of variance (ANOVA) showed that it was not statistically different from the other groups $(p=$ n.s.).

In contrast, a significant effect of diet on the rats' performance was observed during the actual testing procedure. The measure of performance reported here is the ratio of correct to incorrect responses during each block of three sessions (Figure 1). In general, the ratio increased over blocks; however, little or no improvement was observed in the lard20, lard10, or SBO20 groups over testing. The chow-fed animals displayed the best performance, with the difference between groups being more evident during the latter half of the testing period (Blocks 4-6).

These findings were confirmed by ANOVA, in which the chow group was compared with each of the diet groups. These analyses revealed significant group $\times$ block interactions involving the chow group and the lard20 $[F(5,65)=2.85, p<.02], \operatorname{lard} 10[F(5,70)=4.15, p<$ $.002]$, and $\mathrm{SBO} 20[F(5,70)=4.95, p<.001]$ groups. The superior performance of the chow group was further indicated by statistically significant main effects of diet in each of these comparisons (all $p \mathrm{~s}<.05$ ). The SBO5 and chow groups did not differ significantly on either measure $(p s>.05)$.

There were no significant differences between the lard10, lard20, and SBO20 groups on the interaction or main effect measures (all $p \mathrm{~s}>.05$ ). However, there was a significant group $\times$ block interaction for the SBO5 group when compared with the lard 10 group $[F(5,70)=$ $3.22, p<.01]$ and the SBO20 group $[F(5,70)=3.95$, $p<.003$ ]. A comparison of the SBO5 and lard 20 groups revealed a group $\times$ block interaction that approached sig- nificance $[F(5,65)=2.04, p<.08]$. These effects were due primarily to group differences at Blocks 5 and 6 , as determined by a Neuman-Keuls test (all $p s<.05$ ); the main effects of group in these comparisons were not statistically significant (all $p \mathrm{~s}>.05$ ).

\section{DISCUSSION}

The present results extend previous research in demonstrating the deleterious effects of diets high in saturated fatty acids on cognitive function. The effects of a diet high in saturated fatty acids were particularly robust in that varying concentrations of lard did not influence the deficit. A diet consisting of as little as $10 \%(w / w)$ lard impaired conditional discrimination learning as severely as a diet containing twice as much lard. Furthermore, these results demonstrate that diets simply high in overall fat content, in and of themselves, may have adverse effects on cognitive function. That is, the performance of the rats fed the low (5\%) SBO diet was superior to that of the rats fed either of the high-fat diets (20\% lard or SBO), and was virtually indistinguishable from those fed standard laboratory chow. Results from our previous study (Greenwood \& Winocur, 1990) suggested that rats fed laboratory chow performed better than rats fed either the $20 \%$ lard or SBO diets on several tests of learning and memory. The present study now suggests that the low fat content of laboratory chow $(4.5 \% \mathrm{w} / \mathrm{w})$ may be one of the factors influencing the superior performance of rats fed this diet.

Our previous work (Greenwood \& Winocur, 1990) showed that diets high in fat, especially those high in saturated fatty acids, impaired performance on several tests of specific or episodic memory. There was also evidence that lard-fed rats were impaired in mastering a general maze-running skill and in learning a go/no-go responsealternation rule. These results indicate that deficits related to high-fat diets extend to tests of nonepisodic learning and memory, in which prior experience influences performance without the subjects' having to recall the specific experiences. The deficits induced by dietary fat observed in the present study extend the latter finding in that the CDL test made minimal demands on episodic memory, but required the rats to learn an associative rule and organize behavior on the basis of that rule.

The alternation task used in our previous study and the present CDL test share a common feature in that both incorporated a conditional learning component. In each case, the selection of a correct response, whether to press a lever or not (go/no-go alternation), or to press the left or right lever (CDL), was conditional on the presence of a signal. There is considerable evidence, from both the animal (Passingham, Myers, Rawlins, Lightfoot, \& Fearn, 1988; Petrides, 1982) and human (Milner, Petrides, \& Smith, 1985; Petrides, 1985) literatures, that performance on conditional learning tests is mediated by regions within the prefrontal cortex. Complex mazes are not typically used to assess conditional learning, but it is known that damage to the prefrontal cortex disrupts per- 
formance on such tests (Milner, 1965; Thomas \& Weir, 1975). This analysis supports the tentative conclusion that nonspecific learning and memory functions under prefrontal control are susceptible to the effects of diets high in saturated fat. Further research is needed to determine whether other forms of rule learning not dependent on the prefrontal cortex (e.g., discrimination learning) are similarly affected by high-fat diets.

The present results also showed that high levels of both polyunsaturated and saturated fat can impair performance on a CDL task. However, the effects of polyunsaturated fat were related to the amount of SBO in the diet. Rats on a $5 \%$ SBO diet did not differ from chow-fed rats, whereas the $20 \%$ SBO group could not be differentiated from the $10 \%$ or $20 \%$ lard groups. These results are generally consistent with previous evidence (Greenwood \& Winocur, 1990) that showed that both $20 \%$ lard and $20 \%$ SBO diets impaired response-alternation learning more than the chow diet. However, the extent of impairment in the animals fed $20 \%$ SBO was less than that for those fed $20 \%$ lard in our previous work, but was of a similar magnitude in the present study. Our combined evidence, therefore, suggests that polyunsaturated fat can have a negative impact on cognitive function. However, in contrast to saturated fat, in which both $10 \%$ and $20 \%$ lard diets equally impair performance, the effects of polyunsaturated fat are more specific to the nature of the task and the level of fat concentration.

It is important to emphasize that the behavioral impairment observed in the present study cannot be attributed to generalized deficits in attention, motivation, or other performance measures. In order to qualify for the testing phase of this experiment, the rats were required to meet performance criteria in each of three stages of training. The rigorous training program required them to learn to press two levers, adapt to the presence and absence of the levers, and to respond only in the presence of a light stimulus. Only a few rats failed to meet all of the training criteria and they did not disproportionately represent any of the diet groups. Moreover, there were no differences between the diet groups in reaching the criteria in any of the training stages.

As would be expected, the body weights of the rats fed the high-lard or SBO diet were about $20 \%$ higher than those of the chow-fed rats. Obesity can affect expression of motivated behaviors, so it is conceivable that differences in body weight could account for differences between the groups fed the high-fat diets and the other groups. Two points argue against this interpretation. First, as indicated above, obesity was not a factor in reaching the various training criteria in the present study. Second, although the $20 \%$ lard and $20 \%$ SBO groups were equally impaired in the CDL task, behavioral differences between the groups fed identical diets were observed on other learning tasks (Greenwood \& Winocur, 1990). Thus, both the present and previous study suggest that differences between diet groups are linked to specific features of the diets and are unlikely to be explained simply in terms of obesity-related motivational differences.
It has been suggested that diets deficient in EFA, when fed over prolonged periods of time, may impair visual acuity in animals (Neuringer, Anderson, \& Connor, 1988). In view of this, it is conceivable that altered cognitive performance in rats fed EFA-deficient diets may be due, in part, to impaired visual acuity. It is unlikely, however, that altered visual acuity could explain the results of our present experiment. First, our diets were not deficient in EFA and were not fed over the prolonged periods of time used to demonstrate altered visual acuity (Neuringer et al., 1988). Thus, it is unlikely that visual acuity would have been affected to any great extent, if at all. Second, because all the groups progressed through the training stages at an equal rate, it would appear that even if minor alterations in visual acuity were indeed present, they would not have been of a sufficient magnitude to influence the animals' ability to perform the task.

The results of this study raise the question of the mechanism by which dietary fat influences cognitive performance. It is unlikely that the present results can be explained in terms of simple alterations in bulk membrane composition associated with variations in dietary fatty acid composition. Our previous research demonstrated that $20 \%$ lard and $20 \%$ SBO diets fed to rats for as little as 2 months altered neuronal membrane composition (McGee \& Greenwood, 1989). On this basis, a plausible hypothesis was that the alterations in membrane phospholipid fatty acid profile were of a sufficient magnitude to influence neuronal function. For example, previous work has demonstrated alterations in the physiochemical properties of the membrane, membrane-bound protein function, and membrane electrophysiologic properties associated with dietary-fat-induced alterations in membrane lipid composition (for review see Greenwood et al., 1989). Changes in any of these properties could then, in turn, be associated with altered behavior, including cognitive performance.

Following our previous work, one would anticipate different neural membrane compositions in the $20 \%$ lard and $20 \%$ SBO groups of rats in the present study. Nevertheless, there was no measurable difference in performance in the CDL task, suggesting that changes in bulk membrane fatty acid profile were not causally related to the impaired cognitive performance. This apparent dissociation between membrane composition change and behavior is consistent with previous results demonstrating that alterations in feeding behavior induced by dietary fat composition could not be attributed to specific changes in bulk membrane fatty acid composition (McGee \& Greenwood, 1990a). It should be recognized, however, that previous work on neural membranes only measured wholemembrane composition. This technique is not sensitive enough to measure localized differences within the membrane that may have altered the fatty acid composition of annular lipids surrounding specific proteins and may have been of sufficient magnitude to alter specific membrane properties. Thus, our present work cannot be used to entirely rule out the possibility that alterations in neuronal membrane composition is associated with the changes in cognitive performance observed. 
Furthermore, it would appear that the observed deficits in performance cannot simply be attributed to differences in the absolute and/or relative intake of specific fatty acids. The fatty acid profile and content of the $10 \%$ lard, $20 \%$ lard, and 20\% SBO diets differ substantially, but the CDL task performance of the rats fed these diets was indistinguishable. Thus, alterations in the absolute intake of the EFAs C18:2n6 and C18:3n 3 or their ratio in the diet did not account for the differences in cognitive performance observed. This suggests that EFA intake in the absence of EFA deficiency or gross abnormalities in the ratio of the EFA in the diet had little impact on performance outcome. This finding is in contrast to previous reports demonstrating that feeding rats diets that are deficient in EFA, especially during periods of brain development, results in impaired cognitive performance (for review see Greenwood et al., 1989).

Additionally, the overall intake of individual fatty acids and the sum of polyunsaturated, monounsaturated, or saturated fatty acids cannot explain our present results. However, our results do raise the possibility that the saturated fatty acids per se may be involved in the deficit observed. For example, the level of the saturated fatty acids C14:0, C16:0, and $\mathrm{C} 18: 0$ are highest in the 20\% lard diet, almost identical in the $10 \%$ lard and $20 \%$ SBO diets, and lowest in the chow and 5\% SBO diets. Conversely, the performance of the rats fed the $10 \%$ lard and 20\% SBO diets was indistinguishable, yet was poorer than for the rats that consumed the chow and 5\% SBO diets. These results would tend to suggest that the higher intake of saturated fatty acids was associated with poorer outcome. The finding that the performance of the groups fed $10 \%$ lard and $20 \%$ SBO did not differ from that of those fed the $20 \%$ lard may simply reflect the fact that these groups performed at near-chance levels in the CDL test, thereby masking any possible difference between them. This interpretation would be consistent with previous work demonstrating a high correlation between alterations in feeding behavior (protein/carbohydrate selection) and saturated fatty acid composition of the diet (McGee \& Greenwood, $1990 \mathrm{~b})$. A similar argument could be made for the monounsaturated fatty acids $\mathrm{C} 16: 1$ and $\mathrm{C} 18: 1$ but, at present, there is no literature linking the monounsaturated fatty acids to behavioral effects.

Another important dietary variable in this study appeared to be the level of fat fed within the context of a high-polyunsaturated-fat diet. Thus, as carbohydrates replaced fat calories within the $5 \%$ and $20 \%$ SBO diets, there was a beneficial effect on cognitive performance. The rats were in a fasting state during the testing procedure, so the benefits of the low-fat/high-carbohydrate diet cannot be attributed to elevated blood glucose levels associated with immediate meal ingestion. Rather, one would anticipate fasting blood glucose levels to be comparable among all the groups. Nevertheless, these data suggest that the different handling of fat and carbohydrates by the body and their availability as energy substrates to the body may be important variables. Thus, the consumption of low-fat/high-carbohydrate diets might provide the brain with a more accessible and sustainable energy substrate to maintain its daily energy requirements, thereby allowing for better overall brain function. This view is consistent with the argument that dietary fat is not readily oxidized as an energy substrate, especially within the context of a high-fat diet (Flatt, 1978). The idea that the fat/carbohydrate ratio in the diet may be the more important variable is not consistent with the observation that the rats fed $10 \%$ and $20 \%$ lard performed similarly; however, once again, the fact that neither of these groups performed significantly above chance may have masked possible differences in overall cognitive performance.

One dietary factor that could not be controlled for in this experiment was the role (if any) that the intake of the 45-mg Noyes food pellets could play, because the rats from all the dietary treatment groups consumed the Noyes pellets during the testing periods. Because the fat composition of these pellets would be most similar to the chow and SBO diets, they should have had little impact on the overall fat composition of these experimental groups, but may have marginally reduced the overall fat intake of the $20 \%$ SBO group due to a dilutional effect of consuming the low-fat pellets. The consumption of these pellets would have slightly altered the fat composition and overall fat content of the diets fed to the rats consuming the lardbased diets. It should be recognized, however, that the intake of the Noyes food pellets was small compared with the intake of the experimental diets (approximately $15 \mathrm{~g} /$ day) and should not have greatly influenced overall dietary fat patterns. Furthermore, the impairment was observed in the groups in which the Noyes food pellets were most dissimilar from the experimental diets, so it would suggest that any effect that they may have had on dietary fat intake was not of a magnitude sufficient enough to mask the effect of the experimental diets. Ideally, these experiments should be conducted using food pellets that are identical in composition to the experimental diets. Unfortunately, such pellets are not commercially available.

In summary, the results of this study offer further evidence of the deleterious effects of high-fat diets on cognitive performance and suggest that these effects may be related to the level of saturated fatty acid intake. A question that arises from the present research that is also important for understanding the underlying mechanisms relates to whether diets low in overall fat content, but high in polyunsaturated fatty acids, have selective effects on cognitive function. This question is being addressed in a separate study.

\section{REFERENCES}

Bernhart, F. W., \& Tomarelli, R. M. (1969). A salt mixture supplying the National Research Council estimates of the mineral requirement of the rat. Journal of Nutrition, 89, 495-500.

DREYFus P. M. (1988). Vitamins and neurological dysfunction. In J. E. Morley, M. B. Sterman, \& J. H. Walsh (Eds.), Nutritional modulation of neural function (pp. 155-164). New York: Academic Press. DYER, J. D. , \& GREENwOOD, C. E. (1991a). Dietary essential fatty acids change the fatty acid profile of rat neural mitochondria over time. Journal of Nutrition, 121, 1548-1553.

DYer, J. D., \& GreENwOOD, C. E. (1991b). The level of linoleic acid 
in neural cardiolipin is linearly correlated to the amount of essential fatty acids in the diet of the weanling rat. Journal of Nutritional Biochemistry, 2, 477-483.

Dyer, J. D., \& Greenwood, C. E. (1991c). Neural 22-carbon fatty acids in the weanling rat respond rapidly and specifically to a range of dietary linoleic to a-linolenic fatty acid ratios. Journal of Neurochemistry, 56, 1921-1931.

FlaTt, J. P. (1978). The biochemistry of energy expenditure. In G. Bray (Ed.), Recent advances in obesity research (Vol. 2, pp. 211-228). Westport: Technomic.

Foot, M., Cruz, T. F., \& Clandinin, M. T. (1982). Influence of dietary fat on the lipid composition of rat brain synaptosomal and microsomal membranes. Biochemical Journal, 208, 631-640.

Greenwood, C. E., \& Craig, R. E. A. (1987). Dietary influences on brain function: Implications during periods of neuronal maturation. In D. K. Rasin, B. Haber, \& B. Drujan (Eds.), Current topics in nutrition and disease: Vol. I6. Basic and clinical aspects of nutrition and brain development (pp. 159-216). New York: Alan R. Liss.

Greenwood, C. E., McGee, C. D., \& Dyer, J. R. (1989). Influence of dietary fat on brain membrane phospholipid fatty acid composition and neuronal function in mature rats. Nutrition, 5, 278-281.

Greenwood, C. E., \& Winocur, G. (1990). Learning and memory impairment in rats fed a high saturated fat diet. Behavioral \& Neural Biology, 53, 74-87.

McGee, C. D., \& Greenwood, C. E. (1989). Effects of dietary fatty acid composition on macronutrient selection and synaptosomal fatty acid composition in rats. Journal of Nutrition, 119, 1561-1568.

McGee, C. D., \& Greenwood, C. E. (1990a). Dietary fat-induced changes in protein and carbohydrate selection are not explained by alterations in neuronal membrane fatty acid composition. Life Sciences, 47, 933-944.

McGee, C. D., \& GreENwood, C. E. (1990b). Protein and carbohydrate selection respond to changes in dietary saturated fatty acids but not to changes in essential fatty acids. Life Sciences, 47, 67-76.

MiLner, B. (1965). Visually-guided maze learning in man: Effects of bilateral hippocampal, bilateral frontal, and unilateral cerebral lesions. Neuropsychologia, 3, 17-338.
Milner, B., Petrides, M., \& Smith, M. L. (1985). Frontal lobes and the temporal organization of memory. Human Neurobiology, 4, 137-142.

Musten, B., Peace, D. \& Anderson, G. H. (1974). Food intake regulation in the weanling rat: Self-selection of protein and energy. Journal of Nutrition, 104, 563-572.

Neuringer, M., Anderson, G. J., \& Connor, W. E. (1988). The essentiality of $n-3$ fatty acids for the development and function of the retina and brain. In R. E. Olson, E. Beutler, \& H. P. Broquist (Eds.), Annual reviews of nutrition (Vol. 8, pp. 517-541). Palo Alto, CA: Annual Reviews Inc.

Passingham, R. E., Myers, C., Rawlins, J. N. P., Lightfoot, V., \& FEARN, S. (1988). Premotor cortex in the rat. Behavioral Neuro science, 107, 101-109.

Petrides, M. (1982). Motor conditional associative learning after selective prefrontal lesions in the monkey. Behavioral Brain Research, 5 , 407-413.

Petrides, M. (1985). Deficits on conditional associative-learning tasks after frontal and temporal-lobe lesions in man. Neuropsychologia, 23, 601-614.

SANDSTEAD, H. H. (1986). Nutrition and brain function: Trace elements. Nutrition Reviews, 4(Suppl.), 37-41.

Thomas, R. K., \& WeIR, V. K. (1975). The effects of lesions in the frontal or posterior association cortex of rats on maze III. Physiological Psychology, 3, 210-214.

WINOCUR, G. (1991). Functional dissociation of the hippocampus and prefrontal cortex in learning and memory. Psychobiology, 19, 11-20.

Yehuda, S., LeProhon-Greenwood, C. E., Dixon, L. M., \& Coscina, D. V. (1986). Effects of dietary fat on pain threshold, thermoregulation and motor activity in rats. Pharmacology, Biochemistry \& Behavior, 24, 1775-1777.

(Manuscript received February 4, 1993; revision accepted for publication June 25,1993 .) 